\title{
IN SILICO DESIGN OF THE M2 PROTON CHANNEL INHIBITORS OF H1N1 VIRUS
}

\author{
Usman Sumo Friend Tambunan, \\ Mochamad Reza Rahdiansyah and Arli Aditya Parikesit \\ Departement of Chemistry, \\ Faculty Mathematics and Science, University of Indonesia, Depok 16424, Indonesia
}

Received 2012-12-10, Revised 2013-01-17; Accepted 2013-03-15

\begin{abstract}
M2 proton channel of H1N1 Influenza A virus is the target protein anti-flu drugs amantadine and rimantadine. However, the two once powerful adamantane-based drugs lost their $90 \%$ bioactivity because of mutations of virus in recent twenty years. The resistance of influenza A virus to amantadine need to develop more effective adamantane-based drugs. Several research by molecular docking method have been conducted to design and discover ligands which become potential inhibitors for the M2 channel protein of influenza virus in order to inhibit the replication of influenza virus. In this research are studied and evaluated the interaction of ligands towards the protein in the hydrated state using molecular dynamics simulations at two different temperatures. Analysis of ligand interaction yields that AM-L6-R6 ligand has best affinity towards the protein than the T-R6-L6, T-L6-R12 and the standard ligand. It is shown by the ligand interaction on the enzyme active site which remains to be formed during the simulation performed. At the end of simulation temperature of $300 \mathrm{~K}$, AM-L6-R6 ligand has a residue contact with the Arg45 and formed hydrogen bond with Asp44. Then at the end of simulation temperature of $312 \mathrm{~K}$, AM-L6-R6 ligands also could form a hydrogen bond with Asp44. Conformational changes of protein which occur during simulation showed the dynamicization of an protein in the presence of solvent and inhibitor.
\end{abstract}

Keywords: Amantadine, M2 Channel, H1N1 Virus, Molecular Docking, Molecular Dynamics

\section{INTRODUCTION}

Recently, the outbreak of H1N1 influenza A virus is a pandemic of a new strain of influenza virus identified in April 2009 (Trifonov et al., 2009), commonly to as "swine flu". Within only four months, the pandemic has caused many deaths from the first detected country Mexico to almost all countries of the world (http://www.who.int/csr/disease/swineflu/). The H1N1 influenza virus is quite familiar to us because it had caused the 1918-1919 Spain pandemic that had infected $5 \%$ of the world population and resulted in 20-50 million deaths worldwide (Trifonov et al., 2009). In July 2009 the World Health Organization (WHO) enhanced the warning phase 6 , meaning that the spread of H1N1 influenza virus has become a serious global pandemic. The even worse news is that cases were reported that several strains of H1N1 influenza a viruses were resistant to oseltamivir (Tamiflu) (Du et al., 2009).

The M2 channel of the influenza A virus is a homotetrameric protein that contains three different kinds of Transmembrane (TM) four-helix channel with 97 residues per subunit, each of which comprises an intracellular C-terminal domain with a length of 54 residues, a transmembrane domain with a length of 19 residues and an extracellular $\mathrm{N}$-terminal domain with a length of 24 residues (Holsinger and Lamb, 1991; Sugrue and Hay, 1991; Huang et al., 2008; Du et al., 2009; Intharathep et al., 2008). This protein has a major function as a proton-selective channel that is controlled

\section{Corresponding Author: Usman Sumo Friend Tambunan, Departement of Chemistry, Faculty Mathematics and Science,} University of Indonesia, Depok 16424, Indonesia 
by the endosomal $\mathrm{pH}$ values (Shimbo et al., 1996; Huang et al., 2008; Du et al., 2009; Intharathep et al., 2008). In conducting endosomal protons into the virion, the M2 channel is considered to play a crucial role in the viral life cycle (Bright et al., 2006; Wang et al., 2009). Once protons flux into the virion, the uncoating process of the viral nucleic acid in endosomes will be activated, which is essential for the virion to invade the host cells. The $\mathrm{pH}$ regulation of the influenza virus $\mathrm{M} 2$ channel is believed to have strong relationship with the TM fourhelix bundle (Chou et al., 1988; 1992) that is experimentally demonstrated to have proton conductive activity (Duff and Ashley, 1992; Chen et al., 2007). Additionally, this channel has another function to preserve the pre-fusion state of $\mathrm{HA}$ during viral maturation (Wang et al., 2009; Chen et al., 2007; Grambas and Hay, 1992; Takeuchi and Lamb, 1994).

Influenza A virus has the ability to undergo changes by the mechanisms of antigenic drift and shift, resulting new evolving virus strains, which may be extremely toxic and drug resistant (Du et al., 2010; Deyde et al., 2007; Smith et al., 2002). Given that influenza shift may occur every 20-30 years, the danger of future influenza a pandemics highlights the need to develop more effective drugs. The threat of an impending influenza pandemic, possibly through the mutations of the present avian strain $\mathrm{H} 5 \mathrm{~N} 1$ or swine strain $\mathrm{H} 1 \mathrm{~N} 1$, has triggered a global effort to develop more effective antivirus drugs. However, during the past several decades many efforts in developing anti influenza drugs have almost been futile due to the rapid mutations of the influenza virus, resulting in the persistent resistance to the exixting drugs (Du et al., 2010).

Although vaccination is the ideal way to prevent influenza virus infection, the preparation of a new vaccine requires more than 6 months (Couzin-Frankel, 2009). Thus, antiviral drugs are the most effective for short-term defense against influenza. The only known anti-influenza A drugs are M2 (matrix-2-protein) inhibitors (amantadine and its derivative rimantadine) and NA (neuraminidase) inhibitors (zanamivir and oseltamivir) (Hayden and Hay, 1992; Schmidtke et al., 2006). The mechanism of M2 inhibitors is to block the ion channel activity of the M2 protein of most influenza A viruses. This action inhibits viral replication by blocking proton flow. The amino group in amantadine is likely the pharmacophore and is necessary to block proton transport (Hatta et al., 2004; Wang et al., 1993; Betakova, 2007; Cady and Hong, 2008; Tran et al., 2011). Both amantadine and rimantadine are limited in their use in the U.S because of the rapid development of resistance. In addition, there is growing concern that antineuraminidase-resistant viruses may emerge if these drugs are widely used (Lipatov et al., 2004). Thus, there is an urgent need to discover new types of M2 inhibitors for the development of new antiinfluenza drugs. The present study was initiated in an attempt to solve drug resistant problem, to design and screen a small primary amine library of scaffold-hops based on amantadine to generate new hits in the M2 inhibitors class by conducting molecular docking and dynamics method using MOE software (Vilar et al., 2008).

\section{MATERIALS AND METHODS}

The following steps were conducted using Microsoft Windows 7 based PC. Search and choose the sequences (Veits et al., 2012). M2 proton channel sequences were downloaded from Influenza Virus Resource database of the National Center for Biotechnology Information (NCBI) (http://www.ncbi.nlm.nih.gov.html).

\subsection{Multiple Sequence Alignment and Homology Modeling}

This step was conducted by using ClustalW online program (www.ebi.ac.uk/Tools/clustralw2/index.html). Moreover, Homology modeling was performed using the SWISS MODEL which can be accessed through http://swissmodel.expasy.org/SWISS-MODEL.html. The SWISS MODEL service was utilized to build 3D models for $\mathrm{H} 1 \mathrm{~N} 1$ virus protein, by finding the exact templates (Abdussamad and Aris-Brosou, 2011). The PDB files were exposed by using Molecular Operating Environment (MOE) (Vilar et al., 2008).

\subsection{Molecular Docking}

The found 3D structure was docked with its ligand by using molecular docking software. Before the docking, preparation steps must be done. This was done by removing water molecules, addition of hydrogen atoms and charges. Further minimization was done using MMFF94x (Vilar et al., 2008). The utilized parameters for analyzing the complex between protein and ligand are $\Delta \mathrm{G}^{\circ}$ binding and inhibition constant.

\subsection{Molecular Dynamics}

The simulation on protein/ligand complex was done after molecular docking steps with MOE 2008.10. Before molecular dynamics was computed preparation steps were done for molecular docking, followed by inserting the ligand in order to form the protein-ligand complex. Then, the complex was minimized by force field MMFF94x and solvable in Born form. The parameters were utilized in accordance with MOE default, which is ensemble NVT (N, 
total atom; V, Volume; T, Temperature) by using the NPA algorithm. We employed two different temperatures level in this simulation, $300 \mathrm{~K}$ as default MOE temperature and 312 $\mathrm{K}$ for fever temperature. The system was heated up to 312 $\mathrm{K}$ through a constant-volume MD simulation lasting $20 \mathrm{ps}$. The enzyme-inhibitor binding interactions and stability of enzyme-ligand complex were calculated after 5 ns of simulation for all ligands (Tambunan et al., 2010).

\section{RESULTS}

\subsection{M2 Channel Sequence}

M2 channel sequences downloaded from the Influenza Virus Resource database of the National Center for Biotechnology Information (NCBI) (http://www.ncbi.nlm.nih.gov.html). The sequences in NCBI database were downloaded between June 2009 to June 2010. The result give 1203 full-length sequences and 67 protein sequences after collapsing.

\subsection{Multiple Sequence Alignment}

Sequence alignments performed using ClustalW2 program that can be accessed through European Bioinformatics Institute (EBI) (http://www.ebi.ac.uk/Tools/clustalw2/index.html). The downloaded M2 channel FASTA sequences were loaded into ClustalW2 program for multiple alignment process. Sequence alignments were used to see differences of protein sequences and to determine a single representative of $\mathrm{M} 2$ channel that will be targeted. M2 channel sequences was selected >gi|294862152|gb|ACU00946.2| matrix protein 2 [Influenza A virus (A/Denmark/524/2009 (H1N1)) that isolated from human, male sex and 22 years old from Denmark. These sequences submitted on April 19, 2010 by the WHO Collaborating Centre for Reference and Research on Influenza, National Institute for Medical Research, The Ridgeway, Mill Hill, London NW7 1AA, UK (Tambunan et al., 2010; Le and Leluk, 2011).

\subsection{Homology Modeling}

The sequence alignment was performed to determine template for docking simulation. Swiss-model (http://swissmodel.expasy.org/) was employed for template identification for M2 channel (Du et al., 2010; Arnold et al., 2006). The process was conducted by giving sequence file input for browsing the crystal structure in FASTA format. The chosen mode is automated. Based on template identification result, crystal structure of the M2 channel (PDB entry code: 2rlfD) were utilized.
Table 1. Structure of functional groups
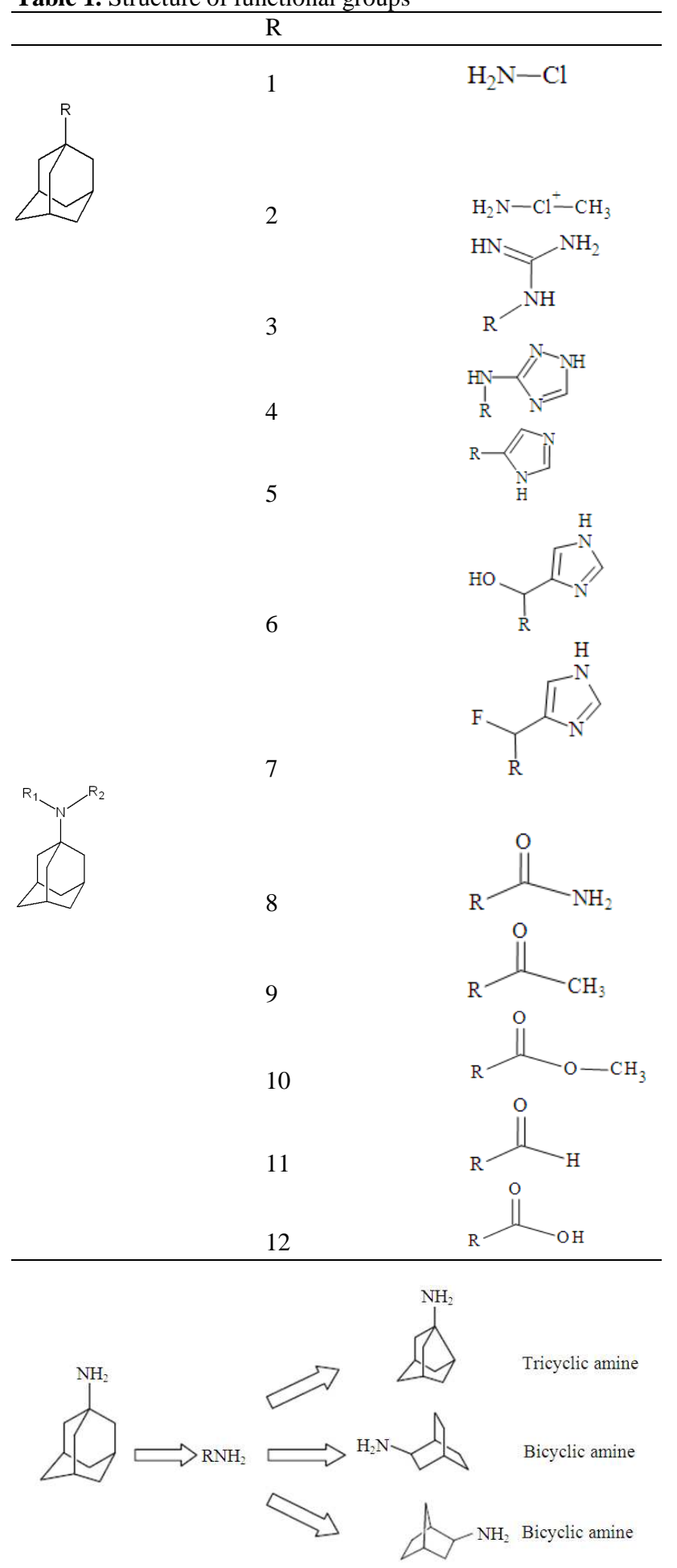

Fig. 1. Design strategy used for the primary amine 


\subsection{Building Ligand Structure}

In this study, 1447 amantadine-based drugs inhibitors have already designed. We designed and screened a small primary amine library of scaffold-hops based on amantadine to generate new hits in the M2 inhibitors class. Among these 1447 compounds, [hydroxy $(1 \mathrm{H}-$ imidazol-4-yl)methyl]tricyclo[3.3.1.03,7]non-3-

ylcarbamic acid (AML6R6), (tricyclo[3.3.1.13,7]dec-1ylimino)bis(1H-imidazol-4-ylmethanol) (TL6R12), (tricyclo[3.3.1.03,7]non-3-ylimino)bis(1H-imidazol-4ylmethanol) (TR6L6) were identified to significantly inhibit M2 channel. The amino group in amantadine is likely the pharmacophore and is necessary to block proton transport (Du et al., 2009). As shown in Fig. 1, the strategy for our library design was based on diversity and the structure-activity relationships of amantadine. The scaffold covers different molecular properties, with an emphasis on steric effect. Herein, we used bicyclic and tricyclic amines. The structures of functional groups are shown in Table 1, to subtitutes pharmacopore group of adamantane-based drugs. The ligand structures were built and minimized optimation by using ACD/PhysChem Suite 12.0. The output is MDL Molfile and then, the output format was converted to MDL Mol format with VEGA ZZ software (Matsubara et al., 2010; Hu et al., 2010).

\subsection{Molecular Docking}

The protein structure was prepared for docking (Pyrkov et al., 2010). This included the addition of missing hydrogens (Gordon et al., 2005). During docking, series of poses (ligand-protein complexes) were generated for each molecule. The quality of each pose was further assessed by the London $\mathrm{dG}(\mathrm{LdG})$ Scoring Function (SF) which estimated the binding free energy of the ligand and a set of the highest score poses were chosen for each molecule (pose) docked (Mazur et al., 2009; Vilar et al., 2008). In this study, MMFF94 and MMFF94x were used for forcefield minimization. MMFF94x was reported as the efficient forcefield for minimizing ligand-protein complexes (Mazur et al., 2009; Kaminski and Jorgensen, 1996; Halgren, 1990). Triangle Matcher (Wildman and Crippen, 2001; 2003; Cook et al., 2010) was applied to orient the ligands in the active site based on charge groups and spatial fit. Triangle Matcher performs a random walk with the ligand in the active site to define the optimal binding orientations. Based on docking simulation result, there are three ligand, namely AML6R6, TR6L6 and TL6R12, could be proposed as a potential inhibitor to the interaction of M2 channel in A/2009(H1N1) virus (Fig. 2).

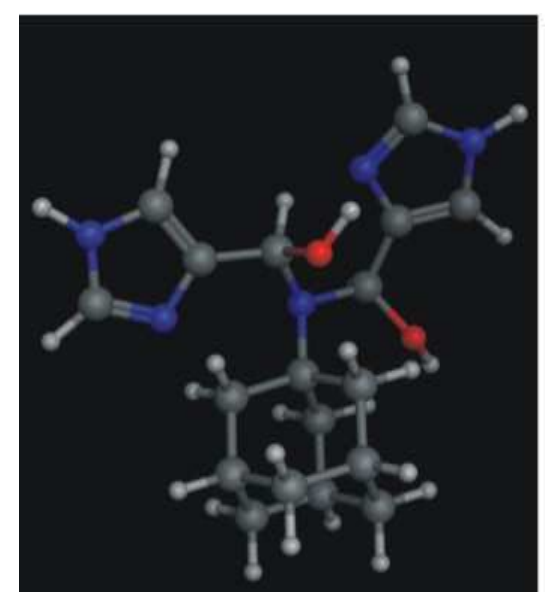

(a)

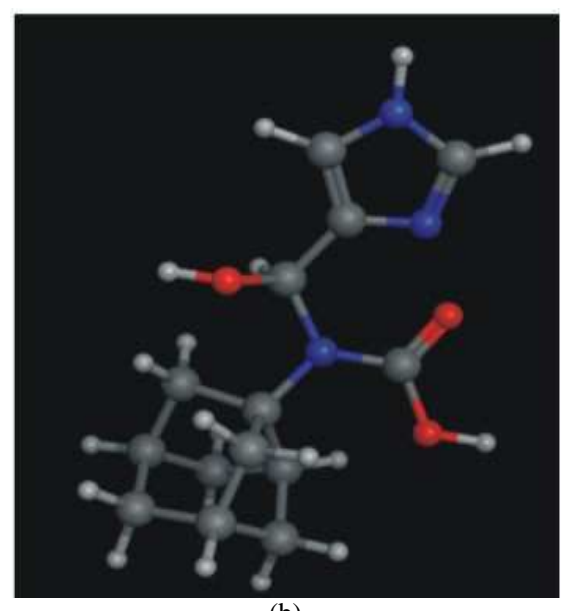

(b)

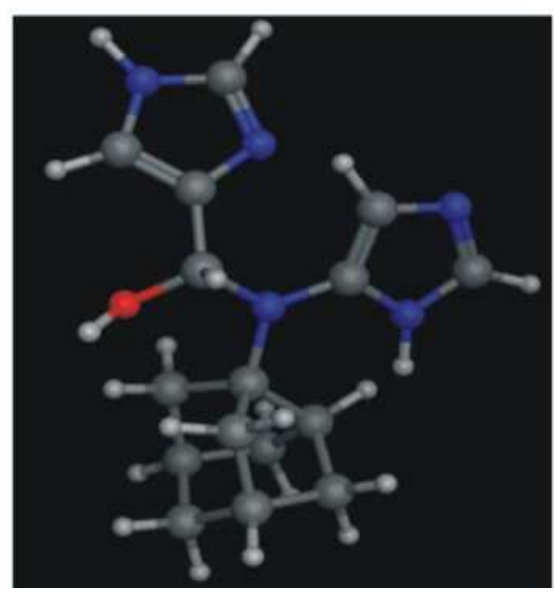

(c)

Fig. 2. The structure of ligands, (a) AML6R6, (b)TL6R12, (c) TR6L6 
In Table 2, it shows the calculated free binding energy ( $\Delta$ Gbind) and hydrogen bond of flexible-ligand docking simulation from the best ligand (Frimurer et al., 2003). Free energy of binding for all the designed ligands were lower than the comparative ligand (amantadine and rimantadine) ( $\mathrm{Du}$ et al., 2010). The free energy of AML6R6, TR6L6, TL6R12, rimantadine and amantadine ligands were negative value, respectivelly, $-6.6766,-$ $5.7709,-4.9738,-4.1198$ and $-3.9574 \mathrm{kcal} / \mathrm{mol}$. The negative and low value of $\Delta \mathrm{G}$ bind indicated the strong favorable bond between enzyme and ligand. In Table 2, we also presented hydrogen bond of AML6R6, TR6L6, TL6R12, rimantadine and amantadine ligands. Bold letters indicate residues of the active site of the M2 channel. The results showed that AML6R6, TR6L6 and TL6R12 binds at channel lock Asp44. Illustrated in Fig. 3-5, ligand TR6-L6 and L6-R12-T form two hydrogen bonds with functioanal residues Asp44. The Asp44 residue is not only the channel lock, but also the protein exit of the M2 channel. Therefore, drug binding at this position not only can hold the channel in the closed conformation but also can simply block the proton exits (Pielak et al., 2009). While the standard ligand amantadine and rimantadine do not form hydrogen bonds with the active site of the M2 channel. Interactions that occur will be validated with the state of the hydrated molecules using molecular

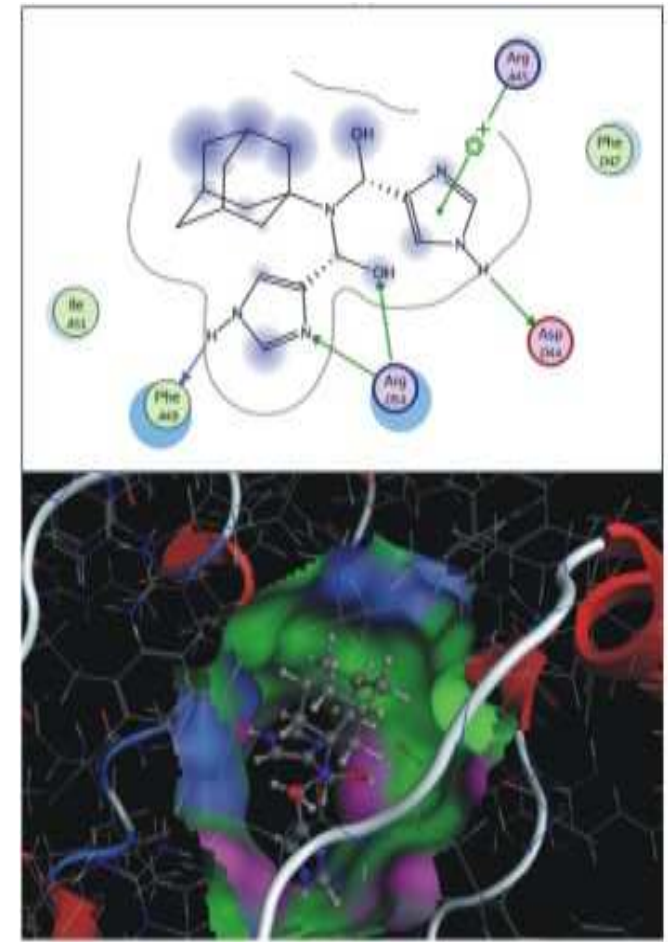

(a) dynamics simulation method (Du et al., 2010; Kumar, 2011; Jing et al., 2008; Zhou and Schulten, 1996).

\subsection{Molecular Dynamics Simulation}

Molecular dynamics simulations are one of the most versatile and widely applied computational techniques for the study of biological macromolecules. They are very valuable for understanding the dynamic behavior of proteins at different timescales, from fast internal motions to slow conformational changes or even protein folding processes (Snow et al., 2005; Alonso et al., 2006). It is also possible to study the effect of explicit solvent molecules on protein structure.

In Table 3, we presented comparison hydrogen bond of AML6R6, TR6L6, TL6R12, rimantadine and amantadine ligands after molecular docking and dynamics simulation. Molecular docking process has resulted all shown ligand to form hydrogen bonds with the functional residues Asp44 (Du et al., 2010). After the simulation conducted, the three ligands are still forming hydrogen bonds with Asp44. AML6R6 and TL6R12 ligands form two hydrogen bonds with Asp44 residue after molecular dynamics simulations (Huang et al., 2008). However TR6L6 ligand losing one hydrogen bond with Asp44 after molecular dynamics simulation process.

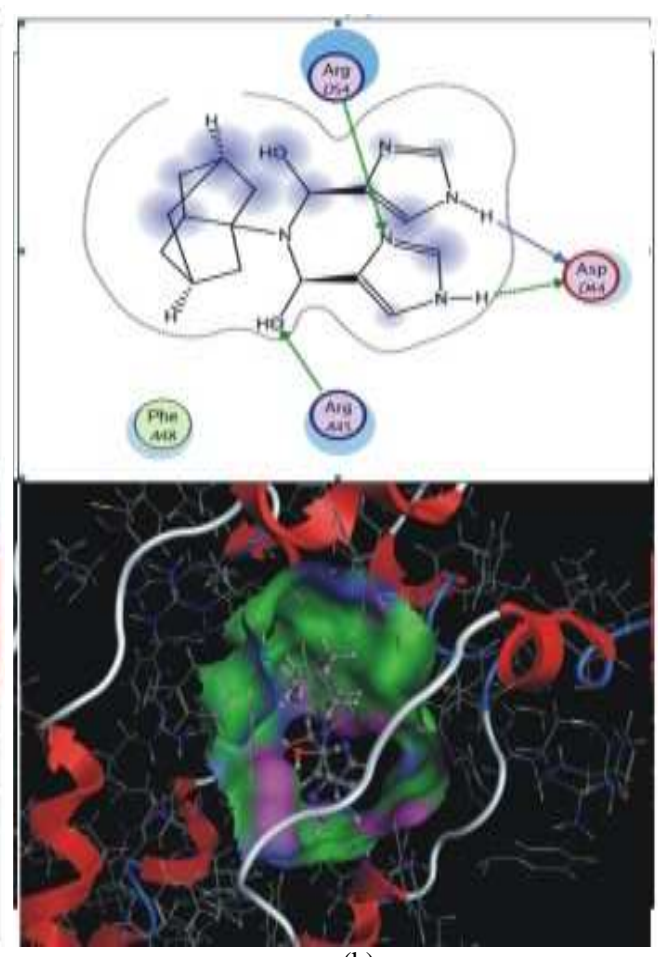

(b) 
Usman Sumo Friend Tambunan et al. / OnLine Journal of Biological Sciences 13 (1): 1-12, 2013

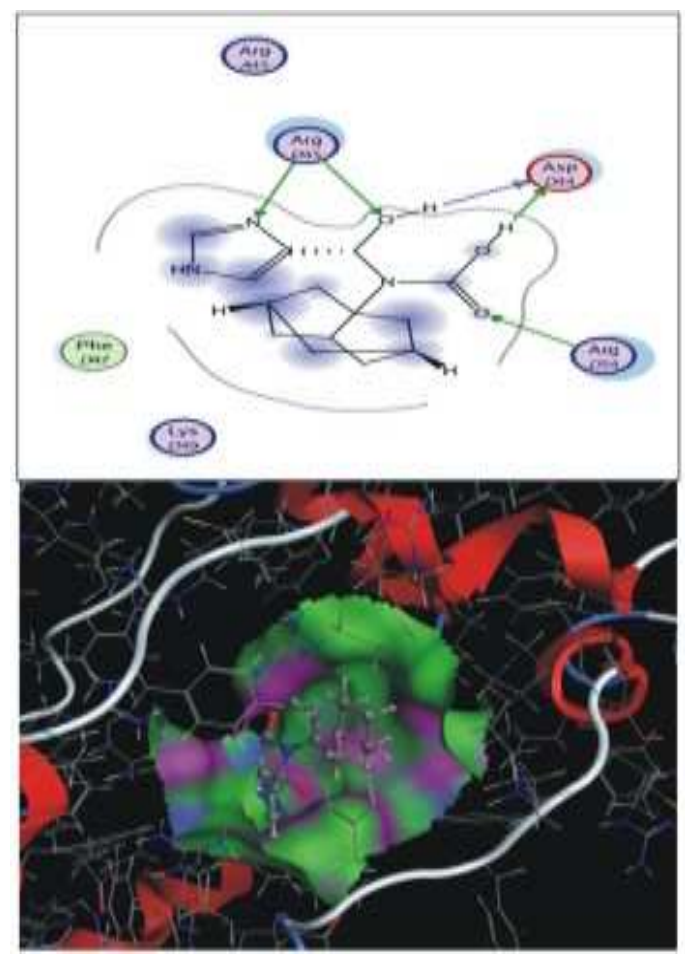

(c)

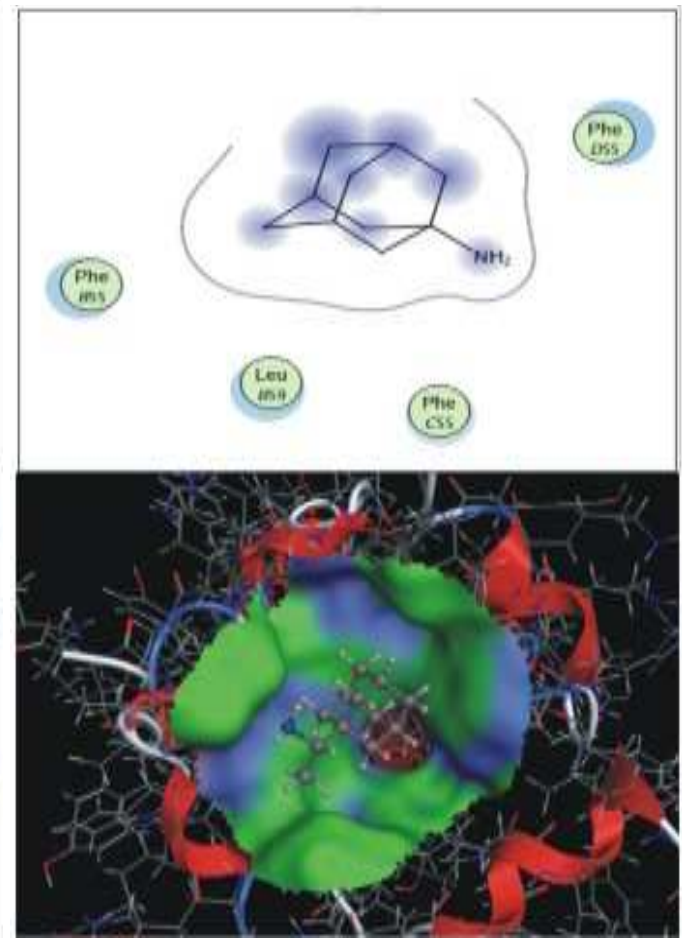

(d)

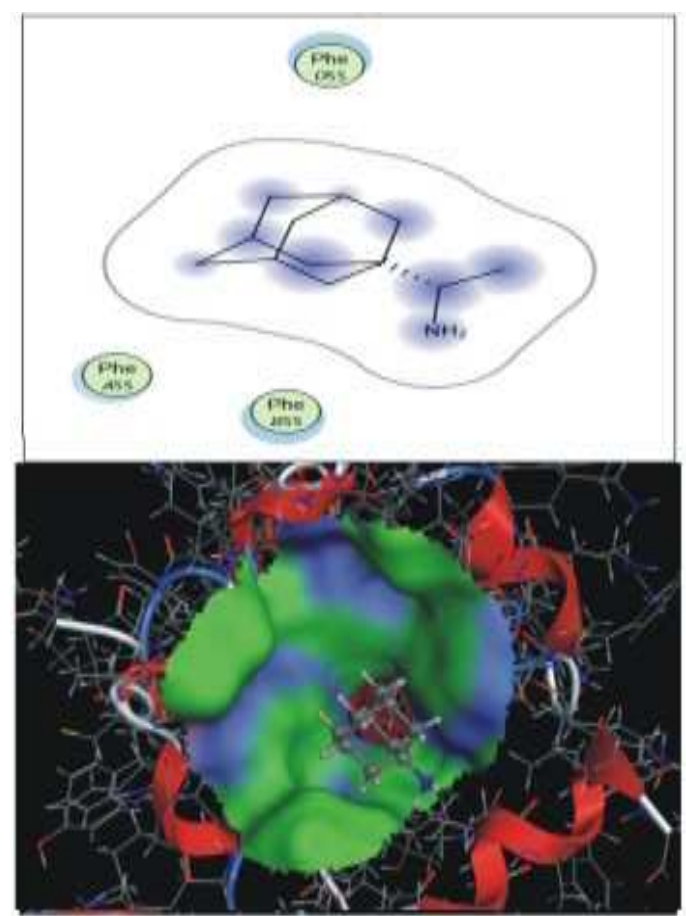

(e)

Fig. 3. Interaction between the M2 channel and ligands (a) AM-L6-R6 (b) T-R6-L6 (c) T-L6-R12 (d) amantadineand (e) rimantadine 
Usman Sumo Friend Tambunan et al. / OnLine Journal of Biological Sciences 13 (1): 1-12, 2013
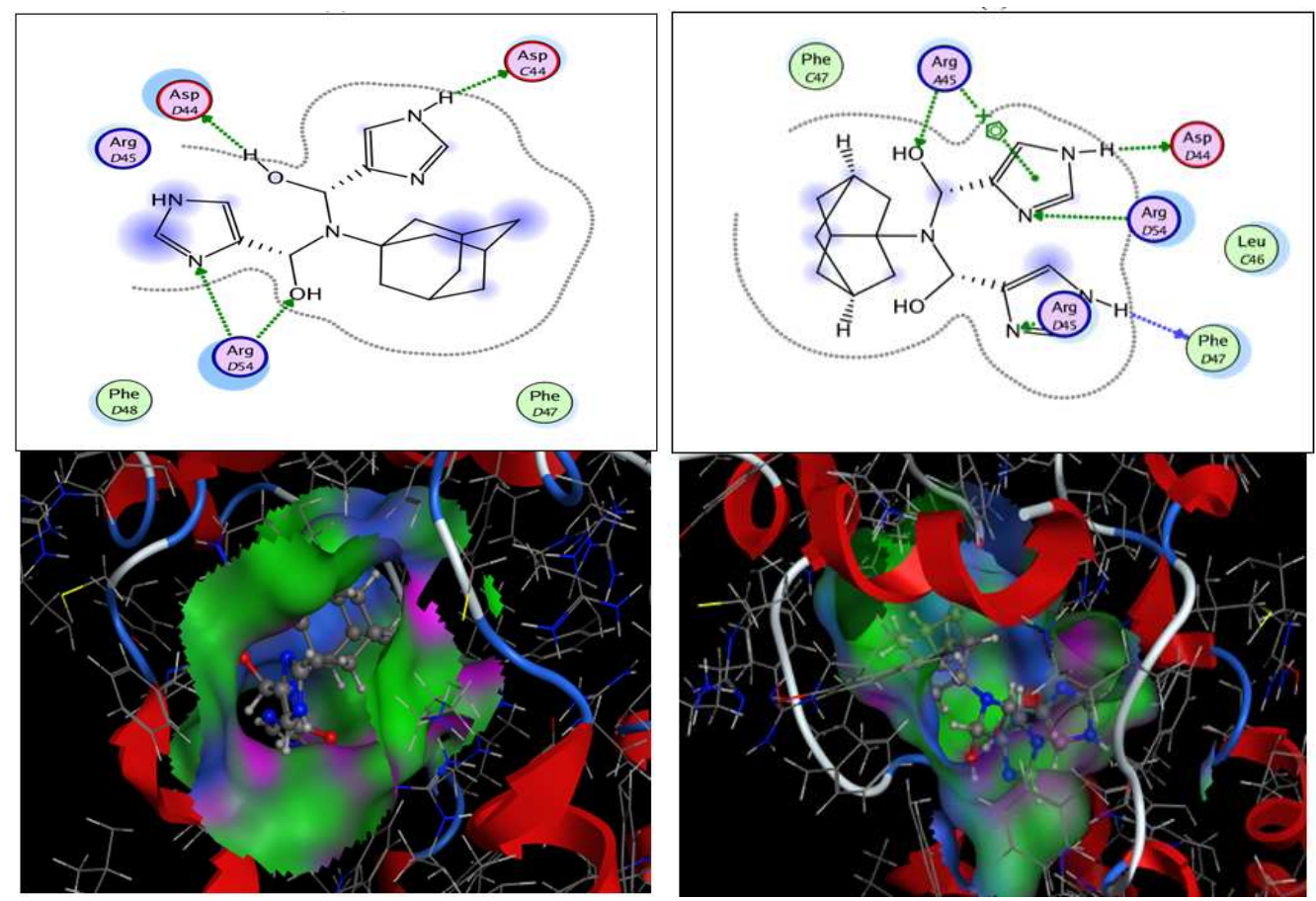

(a)

(b)

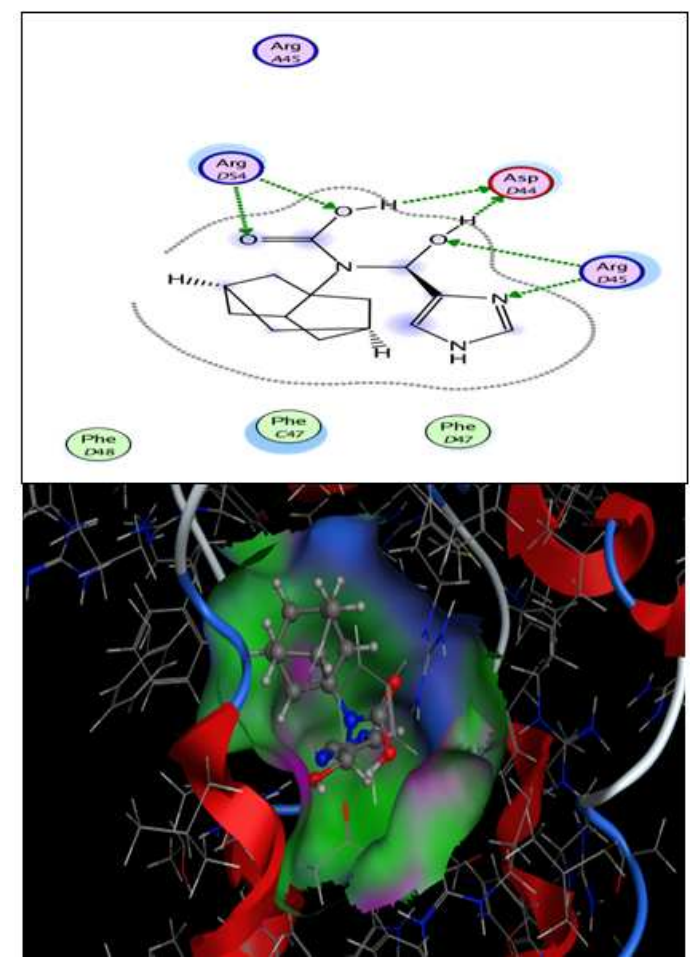

(c)

Fig. 4. Interaction between ligand and the respective amino acid residues in molecular dynamics simulation at 300 K. (a) AML6R6, (b) TR6L6, (c) TL6R12 
Usman Sumo Friend Tambunan et al. / OnLine Journal of Biological Sciences 13 (1): 1-12, 2013

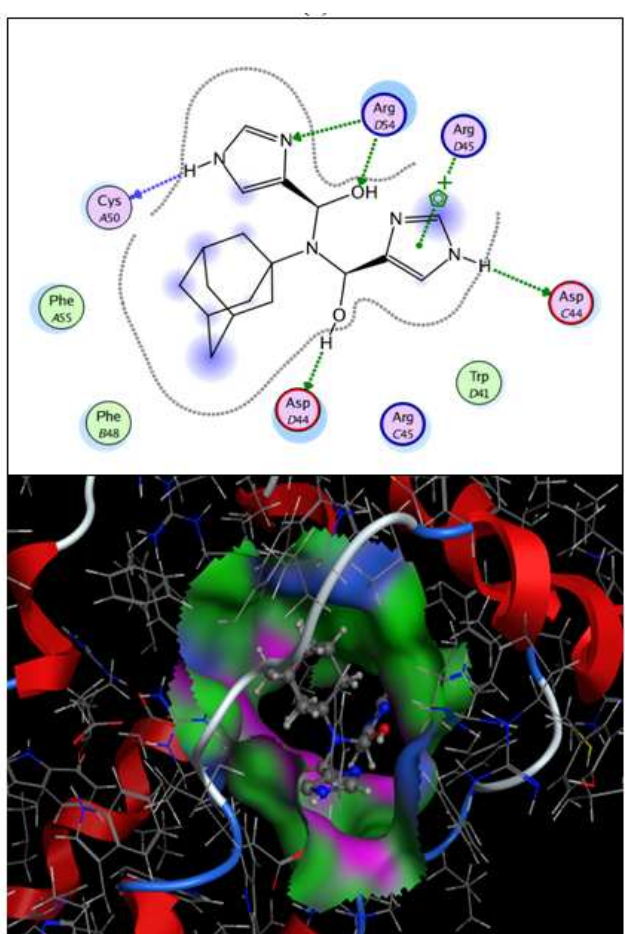

(a)

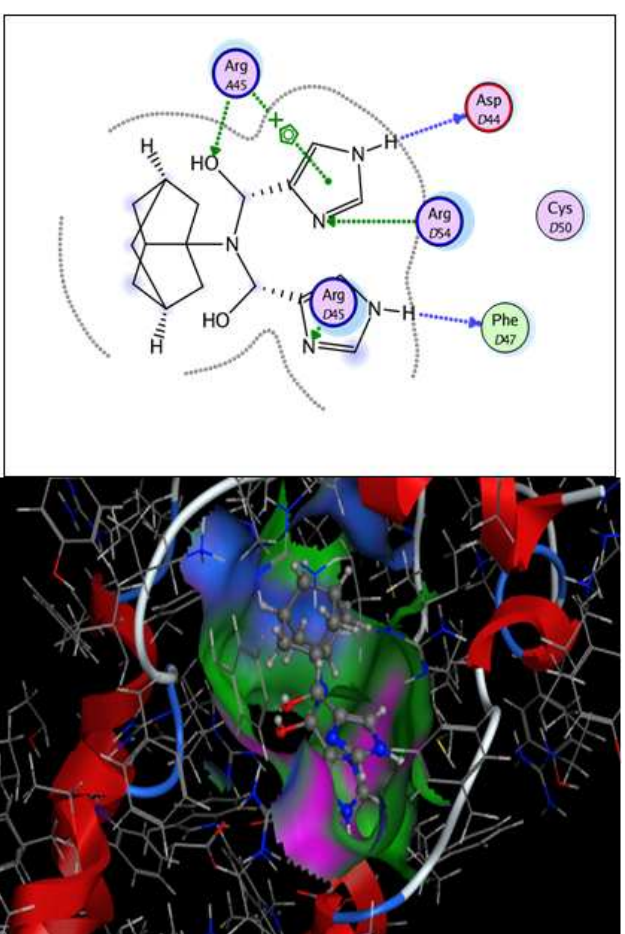

(b)

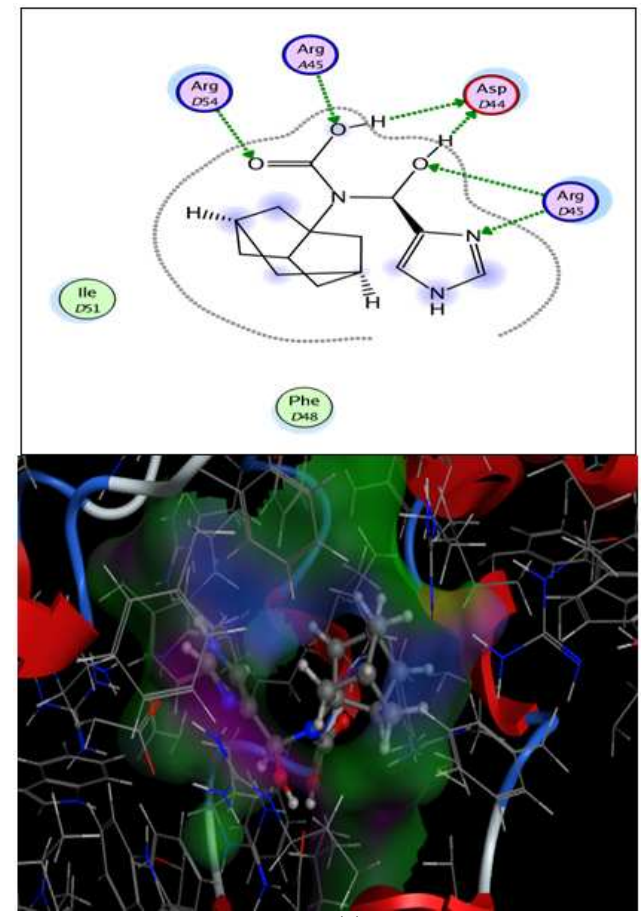

(c)

Fig. 5. Interaction between ligand and the respective amino acid residues in molecular dynamics simulation at $312 \mathrm{~K}$. (a) AML6R6, (b) TR6L6, (c) TL6R12 
Table 2. Binding free energies $\left(\Delta \mathrm{G}_{\text {bind }}\right)$ and hydrogen bond of the best of three ligand

\begin{tabular}{lll}
\hline Ligand & $\Delta \mathrm{G}_{\text {binding }}(\mathrm{Kcal} / \mathrm{mol})$ & Hydrogen bond \\
\hline AM-L6-R6 & -6.6766 & Asp44, Phe48, Arg54, Arg54 \\
T-R6-L6 & -5.7709 & Asp44, Asp44, Arg45, Arg54 \\
T-L6-R12 & -4.9738 & Asp44, Asp44, Arg45, Arg45, Arg54 \\
Rimantadine & -4.1198 & - \\
Amantadine & -3.9574 & - \\
\hline
\end{tabular}

Table 3. Comparison hydrogen bond of the best of three ligand at docking and after dynamics simulation

\begin{tabular}{llll}
\hline Ligan & Docking & Dynamics at 300 K & Dynamics at 312 K \\
\hline AM-L6-R6 & Ile51, Phe47, Asp44, & Arg45, Phe47, Phe48, & Trp41, Arg45, Phe48, Phe55, \\
& Phe48, Arg54, Arg54 & Asp44, Asp44, Arg54, Arg54 & Asp44, Cys50, Arg54, Arg54 \\
T-R6-L6 & Phe48, Asp44, Asp44, & Leu46, Phe47, Asp44, Arg45, & Cys50, Asp44, Arg45, Arg45, \\
& Arg45, Arg54 & Arg45, Phe47, Arg54 & Phe47, Arg54 \\
T-L6-R12 & Arg45, Phe47, Lys49, Asp44, & Arg45, Phe47, Phe47, Phe48, Asp44, & Phe48, Ile51, Asp44, Asp44, \\
& Asp44, Arg45, Arg45, Arg54 & Asp44, Arg45, Arg45, Arg54, Arg54 & Arg45, Arg45, Arg45, Arg54 \\
\hline
\end{tabular}

Residue contact is interaction of M2 channel residue between ligand (inhibitor) (Du et al., 2010). Interaction between complex ligand-M2 channels is formed with hydrogen bond, but also it occurs non-covalent interactions which influence the stability of proteins (Ball and Maechling, 2009). Hydrophobic interactions that cause the protein to maintain its integration because of folding and aromatic interactions that affect the stability of tertiary structure. In Table $\mathbf{3}$ shown AML6R6, TR6L6 and TL6R12 have more residue contacts (italic letter) with M2 channel after molecular dynamics simulations. TL6R12 ligand has more residue contact (Arg45, Phe47, Phe47, Phe48) than two other ligands.

\section{DISCUSSION}

The development of molecular docking and dynamics method has eventually making the drug design workable. The advances of the utilized software are sufficient for generating the necessary ligands and then redirect them for the molecular docking and dynamics computation. Moreover, the pipeline is sufficient for handling this huge amount of data. The time and space complexity of this pipeline are handled by the efficacy of the available graphic subsystem of existing hardware.

Our pipeline could cope with more than 1400 lead compounds, which are much more numerous than what we have before. The screening pipeline was resulted in only three potential inhibitors that perform much better than the standards. Here, the scaling up of our developed pipeline to cope with bigger amount of data seemed to be feasible, provided that the existing hardware and software architecture will support it.
Hydrogen bond, as one of the important chemical bond, is definitely a focal point of this study due its important role for maintaining the interaction between ligand and the protein. The calculation of binding free energy and the related binding sites have eventually exposed important clues on the reactivity of the drugs candidate.

The determination of the molecular dynamics at 300 and $312 \mathrm{~K}$ are indeed useful for simulating the normal and fever human body temperature. The generated data has already sufficient for predicting the stability of the drugs candidate in both temperatures. In this end, a better predictability of the drug mechanism could be achieved by the improved simulation of the temperature.

\section{CONCLUSION}

There are 1447 amantadine-based drugs already designed in this study. The docking of these inhibitors to determine their interaction activities with M2 channel was carried out. The free energy binding $\left(\Delta \mathrm{G}_{\mathrm{bind}}\right)$ values for all the inhibitors were small, indicating the molecules bound considerably well to the binding site. From docking process there are three ligands that have smaller free binding energy ( $\Delta \mathrm{G}_{\text {bind }}$ ) than ligand standard (amantadine and rimantadine) and the best interaction with M2 channel, there are AML6R6, TR6L6 and T-L6-L6-R12. Docking result shown that all ligands form hydrogen bond with functional residue Asp44. After dynamics simulation at $300 \mathrm{~K}$ and $312 \mathrm{~K}$, all ligands still form hydrogen bond with Asp44 residue. To ensure the absorption, distribution, metabolism, excretion and toxicity, ADMET calculations on the ligands are recommended. Thus, from this study, the AML6R6, TR6L6 and TL6R 12 could be proposed as a potential inhibitor to inhibit interaction of M2 channel in H1N1 virus. 


\subsection{Competing Interest}

The researchers declare that they have no competing interests.

\section{ACKNOWLEDGEMENT}

The research was supported by Grant Hibah Bersaing Pasca Sarjana, Indonesian Ministry of Education (Contract Number: 2848/H2.R12/PPM.00 Penelitian/2012). The researchers are grateful to Asif M. Khan, Perdana University, Selangor Darul Ehsan, Malaysia for proof reading the manuscript. USFT supervised this research, MR was working on the technical details and AAP was preparing the English manuscript and re-verified the data.

\section{REFERENCES}

Abdussamad, J. and S. Aris-Brosou, 2011. The nonadaptive nature of the H1N1 2009 Swine flu pandemic contrasts with the adaptive facilitation of transmission to a new host. BMC Evol. Biol., 11: 66. DOI: $10.1186 / 1471-2148-11-6$

Alonso, H., A.A. Bliznyuk and J.E. Gready, 2006. Combining docking and molecular dynamic simulations in drug design. Medic. Res. Rev., 26: 531-568. DOI: 10.1002/med.20067

Arnold, K., L. Bordoli, J. Kopp and T. Schwede, 2006. The SWISS-MODEL Workspace: A web-based environment for protein structure homology modelling. Bioinformatics, 22: 195-201. DOI: 10.1093/bioinformatics/bti770

Ball, V. and C. Maechling, 2009. Isothermal microcalorimetry to investigate non specific interactions in biophysical chemistry. Int. J. Mol. Sci., 10: 3283-3315. DOI: 10.3390/ijms10083283

Betakova, T., 2007. M2 protein-a proton channel of influenza A virus. Curr. Pharm. Des., 13: 32313235. PMID: 18045172

Bright, R.A., D.K. Shay, B. Shu, N.J. Cox and A.I. Klimov, 2006. Adamantane resistance among influenza a viruses isolated early during the 20052006 influenza season in the united states. J. Am. Med. Assoc., 295: 891-894. DOI: 10.1001/jama.295.8.joc60020

Cady, S.D. and M. Hong, 2008. Amantadine-induced conformational and dynamical changes of the influenza M2 transmembrane proton channel. Proc. Nat. Acad. Sci. USA., 105: 1483-1488.
Chen, H., Y. Wu and G.A. Voth, 2007. Proton transport behavior through the influenza A M2 channel: insights from molecular simulation. Biophysical J., 93: 3470- 3479. DOI: 10.1529/biophysj.107.105742

Chou, K.C., G.M. Maggiora, G. Nemethy and H.A. Scheraga, 1988. Energetics of the structure of the four-alpha-helix bundle in proteins. Proc. Nat. Acad. Sci., USA., 85: 4295-4299.

Chou, K.C., G.M. Maggiora and H.A. Scheraga, 1992. Role of loop-helix interactions in stabilizing four-helix bundle proteins. Proc. Nat. Acad. Sci. USA., 89: 73157319.

Cook, I.T., T.S. Leyh, S.A. Kadlubar and C.N. Falany, 2010. Structural rearrangement of SULT2A1: Effects on dehydroepiandrosterone and raloxifene sulfation. Horm. Mol. Biol. Clin. Inves., 1: 81-87.

Couzin-Frankel, J., 2009. What role for antiviral drugs. Science, 324: 705-705. DOI: 10.1126/science.324_705

Deyde, V.M., X. Xu, R.A. Bright, M. Shaw and C.B. Smith et al., 2007. Surveillance of Resistance to Adamantanes among Influenza $\mathrm{A}\left(\mathrm{H}_{3} \mathrm{~N}_{2}\right)$ and $\mathrm{A}\left(\mathrm{H}_{1} \mathrm{~N}_{1}\right)$ Viruses Isolated Worldwide. J. Infect. Dis., 196: 249-257. DOI: $10.1086 / 518936$

Du, Q.S., R.B. Huang, S.Q. Wang and K.C. Chou, 2010. Designing inhibitors of M2 proton channel against H1N1 swine influenza virus.

Du, Q.S., R.B. Huang, C.H. Wang X.M. Li and K.C. Chou 2009. Energetic analysis of the two controversial drug binding sites of the M2 proton channel in influenza a virus. J. Theoretical Biol., 259: 159-164. DOI: 10.1016/j.jtbi.2009.03.003

Duff, K.C. and R.H. Ashley, 1992. The transmembrane domain of influenza A M2 protein forms amantadine-sensitive proton channels in planar lipid bilayers. Virology, 190: 485-489. DOI: 10.1016/0042-6822(92)91239-Q

Frimurer, T.M., G.H. Peters, L.F. Iversen, H.S. Andersen and N.P Moller et al., 2003. Ligand-induced conformational changes: Improved predictions of ligand binding conformations and affinities. Biophys. J., 84: 2273-2281.

Grambas, S. and A.J. Hay, 1992. Maturation of influenza a virus hemagglutinin-estimates of the $\mathrm{pH}$ encountered during transport and its regulation by the M2 protein. Virology, 190: 11-18. DOI: 10.1016/0042-6822(92)91187-Y 
Gordon, J.C., J.B. Myers, T. Folta, V. Shoja and L.S. Heath et al., 2005. H++: A server for estimating $\mathrm{pK}_{\mathrm{a}} \mathrm{s}$ and adding missing hydrogens to macromolecules. Nucl. Acids Res., 33: W368W371. DOI: $10.1093 /$ nar/gki464

Huang, R.B., Q.S. Du, C.H. Wang and K.C. Chou, 2008. An in-depth analysis of the biological functional studies based on the NMR M2 channel structure of influenza a virus. Biochem. Biophys. Res. Commun., 377: 1243-1247. DOI: 10.1016/j.bbrc.2008.10.148

Holsinger, L.J. and R. Alamb, 1991. Influenza virus $\mathbf{M}_{2}$ integral membrane protein is a homotetramer stabilized by formation of disulfide bonds. Virology, 183: 32-43. DOI: 10.1016/0042-6822(91)90115-R

Halgren, T.A., 1990. Maximally diagonal force constants in dependent angle-bending coordinates. II, Implications for the design of empirical force fields. J. Am. Chem. Soc., 112: 4710-4723.

Hayden, F.G. and H.J. Hay, 1992. Emergence and transmission of influenza A viruses resistant to amantadine and rimantadine. Curr. Top. Microbiol. Immunol., 176: 119-130. PMID: 1600749

Hatta, M., H. Goto and Y. Kawaoka, 2004. Influenza B virus requires BM2 protein for replication. J. Virol., 78: 5576-83. PMID: 15140954

Hu, W., S. Zeng, C. Li, Y, Jie and Z. Li et al., 2010. Identification of hits as matrix-2 protein inhibitors through the focused screening of a small primary amine library. J. Med. Chem., 53: 3831-3834. DOI: 10.1021/jm901664a

Intharathep, P., C. Laohpongspaisan, T. Rungrotmongkol, A. Loisruangsin and M. Malaisree et al., 2008. How amantadine and rimantadine inhibit proton transport in the M2 protein channel. J. Mol. Graph. Model., 27: 342-348. PMID: 18620883

Jing, X., C. Ma, Y. Ohigashi, F.A. Oliveira and T.S. Jardetzky et al., 2008. Functional studies indicate amantadine binds to the pore of the influenza A virus M2 proton-selective ion channel. Proc. Nat. Acad. Sci. USA., 105: 10967-72.

Kaminski, G. and W.L. Jorgensen, 1996. Performance of the AMBER94, MMFF94 and OPLS-AA force fields for modeling organic liquids. J. Phys. Chem., 100: 18010-18013. DOI: 10.1021/jp9624257

Kumar, S., 2011. Molecular modeling and identification of substrate binding site of orphan human cytochrome P450 4F22. Bioinformation, 7: 207-210.

Lipatov, A.S., E.A. Govorkova, R.J. Webby, H. Ozaki and M. Peiris, et al., 2004. Influenza: Emergence and control. J. Virol., 78: 8951-8959. DOI: 10.1128/JVI.78.17.8951-8959.2004
Le, L. and J. Leluk, 2011. Study on phylogenetic relationships, variability and correlated mutations in M2 proteins of influenza virus A. PLoS One, 6: e22970-e22970. PMID: 21829678, DOI: 10.1371/journal.pone.0022970

Matsubara, T., A. Onishi, T. Saito, A. Shimada and H. Inoue et al., 2010. Sialic acid-mimic peptides as hemagglutinin inhibitors for anti-influenza therapy. J. Med. Chem., 53: 4441-4449. DOI: 10.1021/jm1002183

Mazur, P., T. Magdziarz, A. Bak, Z. Chilmonczyk and T. Kasprzycka-Guttman et al., 2009. Does molecular docking reveal alternative chemopreventive mechanism of activation of oxidoreductase by sulforaphane isothiocyanates? J. Mol. Mod., 16: 1205-1212. DOI: 10.1007/s00894-009-0628-5

Pielak, R.M., J.R. Schnell and J.J. Chou, 2009. Mechanism of drug inhibition and drug resistance of influenza A M2 channel. Proc. Nat. Acad. Sci., 106: 7379-7384.

Pyrkov, T.V., I.V. Ozerov, E.D. Balitskaya and R.G. Efremov, 2010. Molecular docking: The role of noncovalent interactions in the formation of proteinnucleotide and protein-peptide complexes. Russian J. Bioorganic Chem., 36: 446-455 DOI: 10.1134/S1068162010040023

Sugrue, R.J. and A.J. Hay, 1991. Structural characteristics of the M2 protein of influenza A viruses: Evidence that it forms a tetrameric channel. Virology, 180: 617-624. PMID: 1989386

Shimbo, K., D.L. Brassard, R.A. Lamb and L.H. Pinto, 1996. Ion selectivity and activation of the M2 ion channel of influenza virus. Biophys. J., 70: 13351346. DOI: 10.1016/S0006-3495(96)79690-X

Smith, B.J., J.L. McKimm-Breshkin, M. McDonald, R.T. Fernley and J.N. Varghese et al., 2002. Structural studies of the resistance of influenza virus neuramindase to inhibitors. J. Med. Chem., 45: 2207-2212. PMID: 12014958

Schmidtke, M., R. Zell, K. Bauer, A. Krumbholz, C. Schrader and J. Suess et al., 2006. Amantadine resistance among porcine $\mathrm{H} 1 \mathrm{~N} 1, \mathrm{H} 1 \mathrm{~N} 2$ and $\mathrm{H} 3 \mathrm{~N} 2$ influenza a viruses isolated in Germany between 1981 and 2001. Intervirology, 49: 286-293. PMID: 16809934

Snow, C.D., E.J. Sorin, Y.M. Rhee and V.S. Pande, 2005. How well can simulation predict protein folding kinetics and thermodynamics? Annual Rev. Biophys. Biomolecular Struct., 34: 43-69. DOI: 10.1146/annurev.biophys.34.040204.144447 
Trifonov, V., H. Khiabanian and R. Rabadan, 2009. Geographic dependence, surveillance and origins of the 2009 influenza A (H1N1) virus. N. Engl. J. Med., 361: 115-119. DOI: 10.1056/NEJMp0904572

Tambunan, U.S.F., A. Limanto and A.A. Parikesit, 2010. In silico analysis of hemagglutinin, neuraminidase and matrix 2 of $\mathrm{H} 5 \mathrm{~N} 1$ virus Indonesian strain related to its high pathogenicity. IIOAB J., 1: 17-24

Takeuchi, K. and R.A. Lamb, 1994. Influenza virus M2 protein ion channel activity stabilizes the native form of fowl plague virus hemagglutinin during intracellular transport. J. Virol., 68: 911-919.

Tran, L., S.B. Choi, B.O. Al-Najjar, M. Yusuf and H.A. Wahab et al., 2011. Discovery of potential M2 channel inhibitors based on the amantadine scaffold via virtual screening and pharmacophore modeling. Molecules, 16: 10227-10255. DOI: 10.3390/molecules161210227

Vilar, S., G. Cozza and S. Moro, 2008. Medicinal chemistry and the Molecular Operating Environment (MOE): Application of QSAR and molecular docking to drug discovery. Curr. Top. Med. Chem., 8: 1555-72. PMID: 19075767
Veits, J., S. Weber, O. Stech, A. Breithaupt and M. Graber et al., 2012. Avian influenza virus hemagglutinins $\mathrm{H} 2, \mathrm{H} 4, \mathrm{H} 8$ and $\mathrm{H} 14$ support a highly pathogenic phenotype. Proc. Nat. Acad. Sci. USA., 109: 2579-2584.

Wang, J.F., D.Q. Wei and K.C. Chou, 2009. Insights from investigating the interactions of adamantanebased drugs with the M2 proton channel from the H1N1 swine virus. Biochem. Biophys. Res. Commun., 388: 413-417. PMID: 19665993, DOI: 10.1016/j.bbrc.2009.08.026

Wang, C., K. Takeuchi, L.H. Pinto and R.A. Lamb, 1993. Ion channel activity of influenza A virus M2 protein: Characterization of the amantadine block. J. Virol., 67: 5585-5594.

Wildman, S.A. and G.M. Crippen, 2001. Evaluation of ligand overlap by atomic parameters. J. Chem. Inform. Comput. Sci., 41: 446-450. DOI: 10.1021/ci0000880

Wildman, S.A. and G.M. Crippen, 2003. Validation of DAPPER for 3D QSAR: Conformational search and chirality metric. J. Chem. Inform. Comput. Sci., 43: 629-36. DOI: 10.1021/ci0256081

Zhou, F. and K. Schulten, 1996. Molecular dynamics study of phospholipase A2 on a membrane surface. Proteins, 25: 12-27. PMID: 8727316 